\title{
Relationship between foveal birefringence and visual acuity in neovascular age- related macular degeneration
}

A Weber ${ }^{1,2}$, AE Elsner ${ }^{1}$, M Miura $^{3}$, S Kompa ${ }^{2}$ and MC Cheney

\begin{abstract}
Purpose To investigate the relationship between visual acuity and foveal birefringence in patients with neovascular age-related macular degeneration. Methods In total, 40 patients with choroidal neovascularization underwent macular imaging with scanning laser polarimetry. Bowtie patterns, typically seen in birefringence images of the macula, were evaluated and classified into three categories: (1) regular bowtie present; (2) bowtie present, but disrupted; and (3) no bowtie present. The relation of the bowtie appearance to the best-corrected $\operatorname{logMAR}$ visual acuities was tested (ANOVA).
\end{abstract}

Results Mean visual acuity was best for the group that had regular bowties (mean $\log M A R=0.34)$ and differed statistically significantly from the disrupted bowtie group and no bowtie group $(P=0.01$ and 0.0007$)$. Ages for the three groups did not differ $(P=0.31)$. Conclusions Appearance of a regular bowtie indicates a substantially intact Henle fibre layer with the potential for good visual function, despite the presence of underlying pathology. Conversely, disruption or absence of a bowtie may indicate severe damage to the photoreceptors, consistent with the finding of poorer visual acuity. Eye (2007) 21, 353-361. doi:10.1038/sj.eye.6702203; published online 6 January 2006

Keywords: scanning laser polarimetry; agerelated macular degeneration; visual acuity

\section{Introduction}

Biological materials that have a highly organized spatial arrangement can exhibit birefringence, a meridional variation in refractive index, which differentially retards transmitted light polarized along the 'fast' and 'slow' axes of the material. Consequently, light transmitted through birefringent materials can have dramatically altered polarization characteristics, a property used in polarimetry to examine the structure of birefringent materials. In the normal eye, polarization specific retardation of light occurs mainly in the cornea, which contributes about $80 \%$ of the birefringence of the eye; the remaining birefringence originates in the retinal nerve fibres and the Henle fibres of the central macula. ${ }^{1-3}$ The crystalline lens, tear film, aqueous humour, and vitreous are thought to contribute an insignificant amount to the total ocular birefringence. ${ }^{4,5}$

Scanning laser polarimetry (SLP) has successfully been used to measure thickness of the nerve fibre layer outside the fovea. ${ }^{6-8}$ Also, polarimetry images of the fovea show a characteristic 'bowtie' pattern. ${ }^{9-20}$ This pattern is thought to result from an interaction of corneal and foveal birefringence. The foveal birefringence is fairly uniform, with the birefringent slow axes arranged in a radially symmetric pattern. This pattern is due to the regularly and closely packed axons of the Henle fibre layer. With increasing eccentricity the Henle fibres become less displaced, while there is an increase in the number of ganglion cell axons. ${ }^{21}$

In macular disease, such as in advanced age-related macular degeneration (AMD), this uniform arrangement of the photoreceptors can be disturbed. Photoreceptor loss has been described, for instance in patients with neovascular AMD. ${ }^{22}$ When photoreceptor cell
${ }^{1}$ The Schepens Eye Research Institute and Harvard Medical School, Boston, USA

${ }^{2}$ Department of Ophthalmology, University Hospital, Aachen, Germany

${ }^{3}$ Department of Ophthalmology, Tokyo Medical University, Tokyo, Japan

Correspondence: AE Elsner, Indiana University School of Optometry, 800 E. Atwater, Bloomington, IN 47405, USA Tel: + 1812856 1500; Fax: + 18128557045 E-mail: aeelsner@ indiana.edu

Received: 15 June 2005 Accepted in revised form: 18 October 2005 Published online: 6 January 2006

Disclosure: A. Weber, None; A.E. Elsner, P; M. Miura, None, S. Kompa, None; MC Cheney, None.

This paper was partly presented at ARVO in $\mathrm{Ft}$ Lauderdale in May 2005 
bodies are damaged or die, their axons are likewise affected, leading to disruption of the radially symmetric birefringence of the Henle fibre layer and subsequently to disruption of the typical bowtie pattern. ${ }^{15,20}$

The presence of the bowtie pattern was evaluated in eyes with subfoveal choroidal neovascularization by Katsanos et al to investigate whether choroidal neovascularization (CNV) would influence the measured retardation in SLP. The recently adopted method for minimizing the effect of corneal birefringence requires the macular area to be imaged as the first step in the compensation process. However, the bowtie pattern is lacking in $2 / 3$ of the $\mathrm{CNV}$ cases, indicating that a disrupted macula might not be suited for measurements of corneal birefringence. ${ }^{20}$ Similarly, we show that a bowtie pattern was not seen in a majority of our cases.

In the current investigation we test the hypothesis that the absence or degradation of the bowtie is correlated with loss of foveal function in patients with $\mathrm{CNV}$. We propose that SLP images of the macula could offer a rapid, noninvasive method to acquire objective information about visually significant changes in foveal structure.

\section{Materials and methods}

\section{Patients}

Study participants (19 male/21 female) consisted of a convenient cohort of patients with neovascular AMD, examined in the retina clinic of the ophthalmology department of the University Hospital in Tokyo (Japan), Aachen (Germany) or NORI (Ft Myers, FL, USA) during the time period of February, 2002 through September, 2004. 'Convenient', as previously defined, ${ }^{23}$ implied that every patient during that period who might meet the inclusion criteria was invited to participate by one of the study investigators (MM, SK, AW), if that investigator was present in the clinic and not interacting with other patients. Each eye of a study participant was eligible for enrolment provided that the eye underwent a complete ophthalmologic examination, fluorescein angiography (FA), and SLP during the study visit. If both eyes of a patient had CNV, we selected the eye in which both early and late FA images were ordered. Depending upon patient status, additional diagnostic testing, such as colour fundus photography, indocyanine green angiography, and optical coherence tomography (OCT) was performed. For each patient best-corrected visual acuities were determined and then converted into logarithmic equivalents (logMAR). A group of 14 agematched normal subjects was recruited presenting with visual acuity of at least logMAR 0 .

Written informed consent was obtained from all subjects after explanation of the nature and possible consequences of the study. The protocol was reviewed and approved by the Institutional Review Board of Schepens Eye Research Institute, and following the procedures of the Ethics Committees of the University Hospital in Aachen, Germany, and the University Hospital Tokyo, Japan. Research followed the tenets of the Declaration of Helsinki.

Inclusion criteria were presence of neovascular AMD, with subfoveal, juxta- or extrafoveal $\mathrm{CNV}$, and no prior treatment of the membrane. ${ }^{24,25}$ All eyes had sufficiently clear media for obtaining good quality FA. SLP images were also of good quality. Next, we graded the images from the SLP and the clinical data as follows.

Neovascular lesions were classified according to their angiographic pattern, based on the proportion of classic $\mathrm{CNV}$ and conformed to the definitions previously described for grading and analyzing angiograms. ${ }^{24,25}$ We combined patients with different location and pattern of CNV, which might have influenced VA. The sample size did not allow further analysis of subgroups, but we present the distribution to show the typical diversity of patients with AMD.

\section{Scanning laser polarimetry}

Infrared confocal SLP was performed in all patients with the GDx Nerve Fiber Analyzer (LDT, San Diego, CA, USA). The GDx is a nonmydriatic, noninvasive device that acquires each scan in less than a second. This confocal scanning laser device uses linearly polarized light at $780 \mathrm{~nm}$ to form a $15 \times 15 \mathrm{deg}$ visual angle raster on the retina, for 20 sequential input polarization angles. For image acquisition, the patient's chin rested in the head-and-chin-rest and the patient was asked to fixate either on the centre of the red raster pattern, seen by the test eye, or a target seen by the fellow eye, depending on the patient's respective visual acuities. The instrument was aligned to the patient's cornea and pupil. Then image acquisition was performed three times to obtain good quality images with the fovea approximately centered, equal illumination for all quadrants, and minimal eye movement. For each patient, one image series with sharp retinal vessels and no doubling of the vessels was selected to prevent artefacts from uncorrected eye movements.

Previously, we described our computational approach to SLP, which generates images with different polarization content to emphasize different layers of the retina. ${ }^{26-29}$ Light returning from the retina is captured by two different detectors: the parallel polarized detector that captures the light with the same polarization as the input polarization and the crossed detector, which captures the light that has been rotated by $90^{\circ}$. To compute our images, raw data were first saved, 
consisting of twenty pairs of $256 \times 256,8$ bit greyscale images that vary systematically in illumination polarization angle. A set of Matlab routines (Matlab, Mathworks) then used the raw data to compute a reflectance image from the average amount of light returning to both detectors and a birefringence image from the modulation of light returning to the crossed detector (Figure 1, left and right panel). The reflectance image indicates scan quality and eye position, and the birefringence image provides polarization information about the perifoveal region. Although these two images are routinely provided by the commercial software of the instrument, we used our software to compute the images so that filtering and further alignment to correct for eye movement was available, if needed. The final images were displayed in greyscale (Figures 1-4).

Our GDx contains a fixed birefringent plate in the light path, which is intended to compensate for the birefringence of the cornea. However, since perfect compensation is rare because of the inter-individual variability of both the orientation and the magnitude of the corneal birefringence, ${ }^{11-13,17}$ patients without extensive damage to the Henle fibre layer are expected to have regular bowtie patterns.

Birefringence images computed with our method appear dark since they are generated from only a proportion of the light returning to the crossed detector. For better visualization we enhanced contrast and brightness using the auto level function in Adobe Photoshop 7.0 (Adobe, San Jose, CA, USA) on all image types and did not allow further manipulation of contrast by the operator.

After these computations, three observers (AEE, MCC, AW), masked to the patients' visual acuity and details other than the diagnosis of $\mathrm{CNV}$, separately graded all birefringence images in one session each for presence
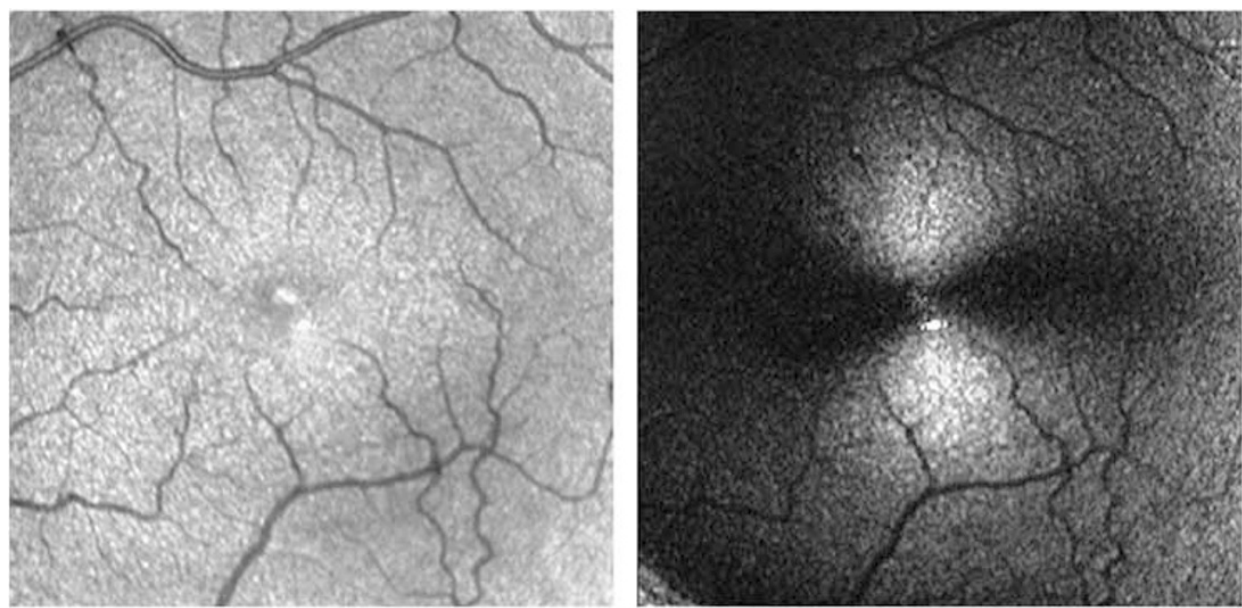

Figure 1 Reflectance image and birefringence image of a 55-year-old female normal subject. Left: Reflectance image showing absence of pathology as expected. Right: Birefringence image showing typical bowtie pattern that is expected due to interaction of corneal and foveal birefringence.
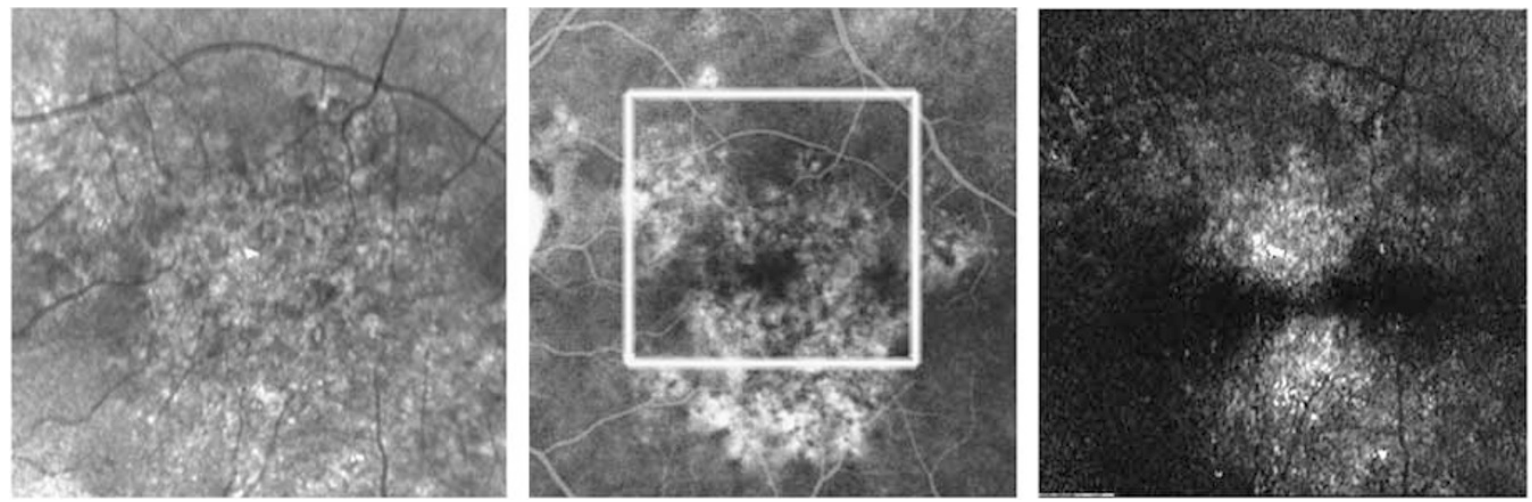

Figure 2 Reflectance image, fluorescein angiogram, and birefringence image of a 65-year-old female patient with subfoveal occult CNV with VA $(\log M A R)$ of 0.10 . Left: Reflectance image showing the lesion and the sharp bordered vessels. This image was used to evaluate image quality. Middle: Late phase FA showing an ill-defined area of stippled hyperfluorescence in accordance with occult CNV. White box indicates area of left and right panels. Right: Birefringence image showing a characteristic regular bowtie pattern. 

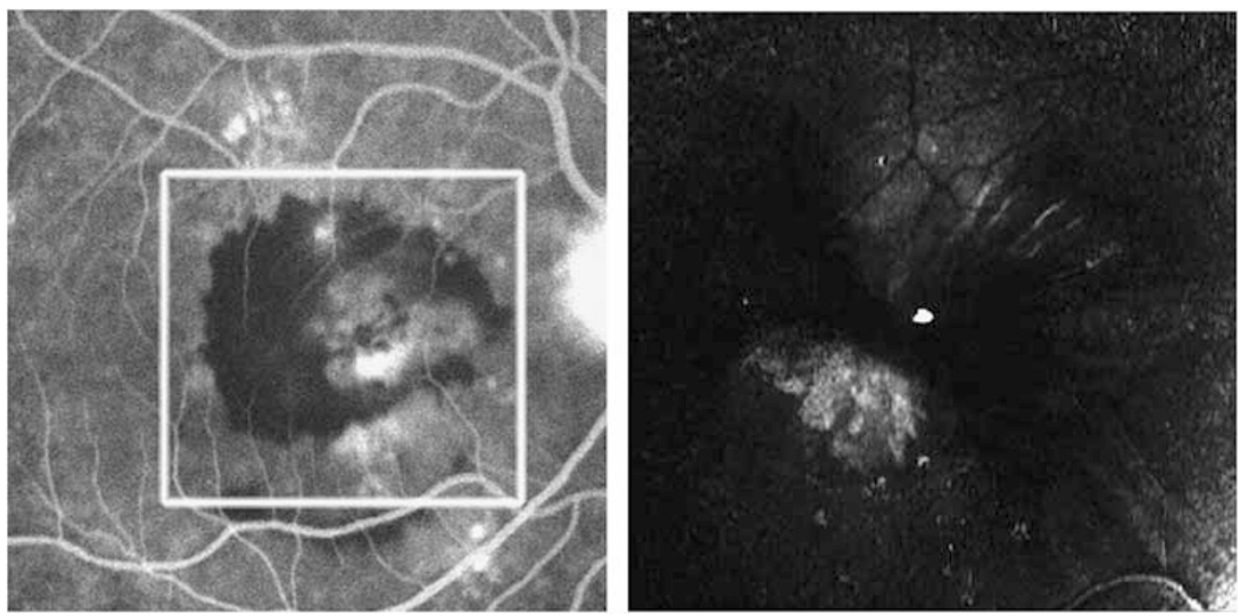

Figure 3 Fluorescein angiogram and birefringence image of a 66-year-old male patient with a minimally classic CNV and VA of 1.1. Left: FA showing a hyperfluorescent area centrally corresponding to the CNV with a large area of fluorescence blockage due to haemorrhage. Right: Birefringence image showing a bowtie with irregular brightness of the arms and additional birefringent structures. Grey borders indicates slight correction for eye movement.
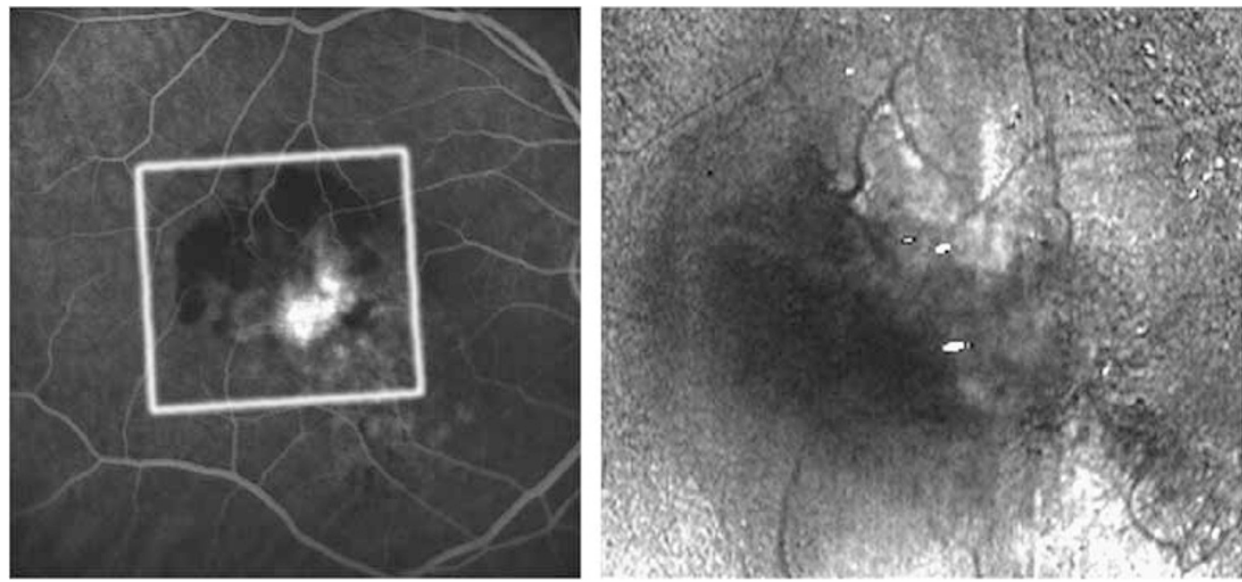

Figure 4 Fluorescein angiogram and birefringence image of a 60-year-old male with a minimally classic lesion. Left: Late phase FA showing juxtafoveal hyperfluorescence of the CNV surrounded by blockage due to haemorrhage. Right: Birefringence image without a characteristic bowtie pattern, but with irregular birefringent structures. Grey borders indicate correction for eye movement.

of the characteristic bowtie pattern. Graders were instructed to grade the birefringence images as follows: a bowtie pattern was categorized as present and regular when it looked as shown in Figures 1 and 2 - a pattern with two white and two dark segments that increased in width with increasing eccentricity and were radially symmetric. To minimize the effect of nerve fibre layer birefringence, which is thought to increase with eccentricity, we graded based on the central part (4 diameter) from the fovea. A disrupted bowtie was present when a bowtie pattern could be identified, but one arm was shaped differently from the opposing arm or when other modulating structures were seen in the bowtie shape (Figure 3). No bowtie was defined as when an image showed random modulation, features unrelated to a bowtie pattern, or a uniform dark background (Figure 4). When there was disagreement about the grading of an image, which occurred in five eyes, the case was discussed among the graders and a final decision was made in order to have only one grade for each case, in a manner similar to that used in previous grading studies of AMD. ${ }^{30,31}$

\section{Statistics}

Analysis of variance was performed using STATVIEW (StatView, Abacus Concepts, Berkeley, CA, USA) to compare ages between the three different bowtie groups and to examine the relation between bowtie classification group and visual acuity. We hypothesised that the group 
with the regular bowtie would have the best visual acuity of the three groups. $P$-values of $<0.05$ were considered statistically significant.

\section{Results}

When separated into three groups according to their bowtie patterns, patients with neovascular AMD differed significantly in their visual acuities $(P=0.0027)$. Mean visual acuity was best in the group with the bowtie present, and was significantly better than the group with a disrupted bowtie and the group without a bowtie $(P=0.01$ and 0.0007$)$, respectively. There was no statistically significant difference between the no bowtie group and the disrupted bowtie group $(P=0.41)$. Mean age, mean visual acuities, distribution of membrane type, and lesion location of the three groups are listed in Table 1. Figure 5 shows logMAR visual acuities for all patients and the distribution for the three different bowtie groups. The groups with AMD did not differ in age $(P=0.31)$. Further, the normal control group did not differ in age compared to the AMD group $(P=0.11)$, but had significantly better VA $(P>0.0001)$ and always presented with regular bowties.

Figure 2 shows an eye with a regular bowtie despite the presence of subfoveal CNV as seen on FA. A total of 10 patients had similar findings. Seven of these eyes had $\log$ MAR visual acuities better than or equal to 0.40 . The remaining three with bowtie present had worse logMAR visual acuities, between 0.7 and 1.0. These three patients had noticeable changes to the inner retina. In two of the three eyes, there were macular neurosensory detachments; and in the other there was occult CNV on FA with abnormal findings in the inner retina on OCT. In two of these cases with poor visual acuity, the CNV was located subfoveally and in the other juxtafoveally. Surprisingly, of the seven cases with better visual acuity, four had subfoveal CNV and one had juxtafoveal CNV with subretinal haemorrhage. Only one had extrafoveal CNV and one juxtafoveal CNV without haemorrhage. Thus, our results indicate that the location of the CNV was not predictive for visual acuity. The type of CNV also was not predictive.

Figure 3 shows an eye with a disrupted bowtie pattern. Of the 12 patients with disrupted bowties, all but two had poor VA's, ranging from 0.52 to 1.4. These two patients with good visual acuity $(-0.08$ and 0.22 logMAR VA) had occult CNV, one had a pigment epithelial detachment. Again, the location of the CNV did not predict visual acuity, since two eyes with CNV outside the fovea had poor visual acuity, whereas two eyes with subfoveal CNV had better visual acuity (Table 1, Figure 5).

Figure 4 shows an eye with no bowtie present. All 18 patients without a bowtie had logMAR VA worse than 0.40. In five of these cases, CNV was not located subfoveally (Table 1), but subretinal haemorrhage extended underneath the fovea. In 16 eyes without a bowtie, various birefringent structures were seen that often corresponded to pathology.

\section{Discussion}

In this study we investigated the effects of neovascular AMD on foveal birefringence by assessment of the bowtie pattern seen on macula images in SLP. It is remarkable that despite severe neovascular changes, many patients had a sufficiently intact Henle fibre layer to produce an intact bowtie pattern or at least a disrupted

Table 1 Mean age, mean visual acuity and distribution of lesion type and lesion location of the 40 patients with neovascular AMD and the age-matched normal subjects

\begin{tabular}{lcccc}
\hline & Present, complete bowtie & Present, disrupted bowtie & Absent bowtie & Normal subjects \\
\hline Number of cases & 10 & 12 & 18 & 14 \\
Mean age & $67 \pm 7$ & $67 \pm 13$ & $72 \pm 10$ & $63 \pm 8$ \\
Mean VA (logMAR) ${ }^{\mathrm{a}}$ & $0.34 \pm 0.4$ & $0.85 \pm 0.4$ & & $-0.03 \pm 0.04$ \\
& & & 7 & \\
Lesion type & 4 & 6 & 9 & 2 \\
$\quad$ Predominantly & 2 & 4 & & \\
$\quad$ Classic & 4 & 2 & & \\
Minimally classic & & & 4 \\
Occult & & 10 & 1 \\
Lesion location & 6 & 1 & \\
$\quad$ Subfoveal & 4 & 1 & \\
Juxtafoveal & 0 & & & \\
Extrafoveal & & & & \\
\hline
\end{tabular}

${ }^{a}$ Visual acuity in logarithm of minimal angle of resolution. 
one. Further, our results show a close relation between appearance of a bowtie pattern and visual acuity in patients with neovascular AMD. Mean visual acuities in the patient group that had a complete macular bowtie were significantly better than those for the other two groups.

About one quarter of our sample had a structure that we classified as disrupted bowtie. The presence of structures that resemble the typical bowtie implies that the Henle fibre layer still seems to work as an intraocular polarimeter, despite tissue disruption due to neovascularization. However, these bowties show asymmetric retardation or partial disruption of this structure that is not directly related to the normal foveal or corneal birefringence. At least a portion of the bowtie was seen in these patients who had a mean visual acuity of 0.85 , which is significantly worse than mean visual acuity for the regular bowtie group matched in age. Although corneal birefringence can change the orientation of the bowtie pattern, or its amplitude, a disrupted bowtie does not result from individual differences in the cornea because the light scanned onto, and returning from the retina, passes through a large and fairly constant area of the cornea. ${ }^{9}$ Instead, this pattern is due to light tissue interactions in the retina.

In 18 of 40 cases we did not identify a bowtie pattern. In a situation with perfect compensation of corneal birefringence, a uniform polarization pattern would be seen instead of a bowtie pattern. ${ }^{9}$ For our analysis, we used an instrument with a fixed-angle compensation method, which assumes all individuals to have a fixed slow corneal axis of $15^{\circ}$ nasally downward and retardation of $60 \mathrm{~nm}$. Newer models of the GDx have a variable compensator that typically produces a macular

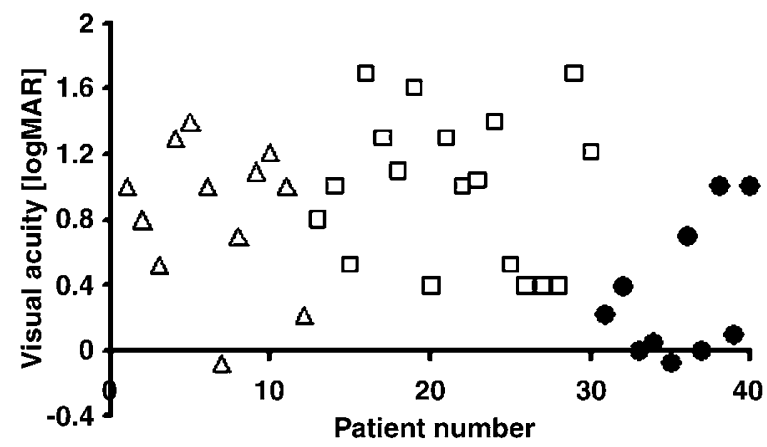

Figure 5 Scatter plot showing the visual acuities of the patients grouped by their bowtie appearance. Note the large proportion of patients with good visual acuity in the regular bowtie group, although there is some overlap in VA of patients from groups. Consistently poor visual acuities occurred in the patients without a well-formed bowtie (squares), and largely poor visual acuities for the disrupted bowtie group (triangles). Squares - no bowtie; triangles - disrupted bowtie; circles - regular bowtie. bowtie until the compensator is adjusted to minimize the corneal birefringence. Previous studies have shown that corneal polarization axes vary widely among normals; ${ }^{13,17}$ in a study with 118 eyes of 63 healthy patients, only one-third of the corneas had a corneal polarization axis of 11 to $20^{\circ}$ nasally downward. These compensated eyes would have weak or no bowties, with the remaining two thirds of eyes presumably having quite visible bowties. Similarly, corneal polarization magnitude was shown to have great inter-individual variation, ${ }^{11,17}$ so that it is unlikely that in our sample, in which nearly half of the patients had no bowtie, perfect corneal compensation was reached. In our patient group, the axis of orientation was not a variable, and only 10 of 40 patients had a regular bowtie. This was too few to permit a quantitative result for the characterization of polarization axis. Most of our cases not only lacked the bowtie spatial pattern but also showed spatially localized regions of birefringence, indicating polarizing properties of pathologic structures, such as fibrovascular tissue, haemorrhage, or exudates (Figure 4). A uniform polarization pattern, which resembles those shown in perfectly compensated corneas, was only seen in two eyes. For this reason we hypothesize that only a few, if any, cases had perfect compensation for corneal birefringence and that the lack of a bowtie might indicate severe structural changes due to the underlying pathology. This hypothesis is also supported by the fact that the patients lacking bowties had worse mean visual acuity compared to the mean visual acuity in the patients with bowties.

Although AMD with neovascularization is a leading cause of blindness in the Western world, the natural history of neovascular complications and visual acuity is variable and difficult to predict. ${ }^{32-35}$ Disease progression has been discussed in terms of individual differences in location and lateral extent of a neovascular lesion, and also in associated changes of the choriocapillaris, Bruch's membrane and retinal pigment epithelium. ${ }^{36}$ Some of our cases had juxtafoveal or extrafoveal location of the CNV. However, that does not mean that the fovea was unaffected, since seven of 40 patients in our sample had poor visual acuity and a disrupted or no bowtie despite a juxta- or extrafoveal location of the membrane. Some cases had subretinal haemorrhage extending under the fovea. Bynoe et $a l^{37}$ showed that neovascular membranes can extend laterally more than expected based on FA because there can be an avascular component. ${ }^{37}$ Both an avascular component or subfoveal haemorrhage could lead to poor visual acuity when the photoreceptor/RPE complex in the fovea is affected. The photoreceptor axons, independent of photopigment or wave-guide mechanisms, are what our method primarily tested, since 
the foveal birefringence is due to the radial order of the photoreceptor axons. ${ }^{38-41}$

For good visual performance, the status of the inner retina and ocular media is important as well. Only three cases in our sample presented with poor visual acuity and a regular bowtie. On OCT two of these cases were shown to have neurosensory detachments, and the other had high reflectivity in the inner retina. In these three cases the unexpectedly poor visual acuity may be due to structural alteration in the inner retinal elements superficial to the photoreceptors, which is not expected to affect the bowtie in the fovea. In patients within this age range, there is a wide variety of diseases that affect the inner retina, such as epiretinal membrane, and when amenable to treatment there is the potential for improvement of visual acuity. ${ }^{42-44}$ In older patients, cataract also degrades visual acuity. Although we did not explore the effect of cataracts, the poor visual acuity in these three patients is unlikely to be explained by cataract, since we required sufficiently clear media to allow good quality FA. We did not evaluate fixation of the patient, so that we cannot rule out foveal vs extrafoveal fixation, leaving uncertain which part of the retina subserved the visual acuity task. However, our reflectance images, digitized from the same data series as the birefringence images, clearly visualized the central macular features of these patients.

Taken together these results imply that the presence of a bowtie may indicate the potential for good visual prognosis, based on outer retinal function, but only in the presence of a functioning visual system. To test visual function, we used visual acuity, which does not fully describe visual function, but yet is commonly used as a rapid assessment of macular function. ${ }^{24,25,45}$

Nevertheless, to obtain good visual acuity and central macula function densely packed and intact photoreceptor axons are needed and this is what we measured with this method.

Foveal birefringence is used increasingly in polarized light techniques for evaluating the eye in a noninvasive and objective manner. The newer version of the GDx employs foveal birefringence to determine corneal birefringence and compensate for it, thereby improving the accuracy of nerve fibre layer measurements. ${ }^{16,46-49}$ Foveal birefringence has also been used for automatic detection of foveal fixation. ${ }^{50}$ Moreover, it is thought that foveal birefringence contributes to Haidinger's brushes, an entoptic phenomenon involving the preferential absorption of short-wavelength light by macular pigment. ${ }^{51-54} \mathrm{We}$ used foveal birefringence to investigate the structure of foveal photoreceptors axons, finding a close relation in most patients between visual function and appearance of foveal polarization images.

\section{Acknowledgements}

We thank the Retina Consultants of SW Florida for their assistance with patient recruitment and Dr Arthur Bradley and Celeste Biever for their reading of our manuscript. This work was supported by National Eye Institute grant EYO7624 to AEE.

\section{References}

1 Van Blokland GJ, Verhelst SC. Corneal polarization in the living human eye explained with a biaxial model. J Opt Soc Am A 1987; 4: 82-90.

2 Van Blokland GJ. Ellipsometry of the human retina in vivo: preservation of polarization. J Opt Soc Am A 1985; 2: 72-75.

3 Brink HB, van Blokland GJ. Birefringence of the human foveal area assessed in vivo with Mueller-matrix ellipsometry. J Opt Soc Am A 1988; 5: 49-57.

4 Weale RA. Sex, age and the birefringence of the human crystalline lens. Exp Eye Res 1979; 29: 449-461.

5 Bueno JM, Campbell MC. Polarization properties of the in vitro old human crystalline lens. Ophthalmic Physiol Opt 2003; 23: 109-118.

6 Weinreb RN, Dreher AW, Coleman A, Quigley H, Shwa B, Reiter K. Histopathologic validation of Fourier-ellipsometry measurements of retinal nerve fiber layer thickness. Arch Ophthalmol 1990; 108: 557-560.

7 Morgan JE, Waldock A, Jeffrey G, Cowey A. Retinal nerve fiber layer polarimetry: histological and clinical comparison. Br J Ophthalmol 1998; 82: 684-690.

8 Dreher AW, Reiter K, Weinreb RN. Spatially resolved birefringence of the retinal nerve fiber layer assessed with a retinal ellipsometer. Appl Opt 1992; 31: 3730-3749.

9 Zhou Q, Weinreb RN. Individualized compensation of anterior segment birefringence during scanning laser polarimetry. Invest Ophthalmol Vis Sci 2002; 43: 2221-2228.

10 Knighton RW, Huang XR, Greenfield DS. Analytical model of scanning laser polarimetry for retinal nerve fiber layer assessment. Invest Ophthalmol Vis Sci 2002; 43: 383-392.

11 Knighton RW, Huang XR. Linear birefringence of the central human cornea. Invest Ophthalmol Vis Sci 2002; 43: 82-86.

12 Greenfield DS, Knighton RW. Stability of corneal polarization axis measurements for scanning laser polarimetry. Ophthalmology 2001; 108: 1065-1069.

13 Greenfield DS, Knighton RW, Huang XR. Effect of corneal polarization axis on assessment of retinal nerve fiber layer thickness by scanning laser polarimetry. Am J Opthalmol 2000; 129: 715-722.

14 Choplin NT, Zhou Q, Knighton RW. Effect of individualized compensation for anterior segment birefringence on retinal nerve fiber layer assessments as determindes by scanning laser polarimetry. Ophthalmology 2003; 110: 719-725.

15 Bagga H, Greenfield DS, Knighton RW. Scanning laser polarimetry with variable corneal compensation: identification and correction for corneal birefringence in eyes with macular disease. Invest Ophthalmol Vis Sci 2003; 44: 1969-1976.

16 Bagga H, Greenfield DS, Feuer W, Knighton RW. Scanning laser polarimetry with variable corneal compensation and optical coherence tomography in normal and glaucomatous eyes. Am J Ophthalmol 2003; 135: 521-529. 
17 Weinreb RN, Bowd C, Greenfield DS, Zangwill LM. Measurement of the magnitude and axis of corneal polarization with scanning laser polarimetry. Arch Ophthalmol 2002; 120: 901-906.

18 Weinreb RN, Bowd C, Zangwill LM. Glaucoma detection using scanning laser polarimetry with variable corneal polarization compensation. Arch Ophthalmol 2003; 121 218-224.

19 Garway-Heath DF, Greaney MJ, Caprioli J. Correction for the erroneous compensation of anterior segment birefringence with the scanning laser polarimeter for glaucoma diagnosis. Invest Ophthalmol Vis Sci 2002; 43: 1465-1474.

20 Katsanos A, Kothy A, Papp A, Hollo G. Influence of subfoveal choroidal neovascularisationon macular imaging with scanning laser polarimetry of the retinal nerve fibre layer. Eye 2005; 19: 117-122.

21 Vrabec F. The temporal raphe of the human retina. Am J Ophthalmol 1966; 62: 926-938.

22 Green WR, Enger C. Age-related macular degeneration histopathologic studies. Ophthalmology 1993; 100: 1519-1535.

23 Brown JC, Solomon SD, Bressler SB, Schachat AP, Di Bernado C, Bressler NM. Detection of diabetic foveal edema. Arch Ophthalmol 2004; 122: 330-335.

24 Macular Photocoagulation Study Group. Laser photocoagulation of subfoveal neovascular lesions in agerelated macular degeneration. Results of a randomized clinical trial. Arch Ophthalmol 1991; 109: 1220-1231.

25 TAP Study Group. Photodynamic therapy of subfoveal choroidal neovascularisationin age-related macular degeneration withVerteporfin: one-year results of two randomized clinical trials-TAP report 1. Arch Ophthalmol 1999; 117: 1329-1345.

26 Elsner AE, Miura M, Stewart JB, Kairala MBM, Burns SA. Novel algorithms for polarization imaging resulting in improved quantification of retinal blood vessels. Stud Health Technol Inform 2003; 94: 59-61.

27 Burns SA, Elsner AE, Mellem-Kairala MB, Simmons RB. Improved contrast of subretinal structures using polarization analysis. Invest Ophthalmol Vis Sci 2003; 44: 4061-4068.

28 Weber A, Cheney MC, Smithwick QYC, Elsner AE. Polarimetric imaging and blood vessel quantification. Opt Express 2004; 12: 5178-5190. http:/ / www.opticsexpress.org/ abstract.cfm?URI = OPEX-12-21-5178.

29 Mellem-Kairala MB, Elsner AE, Weber A, Simmons RB, Burns SA. Improved contrast of peripapillary hyperpigmentation using polarization analysis. Invest Ophthalmol Vis Sci 2005; 46: 1099-1106.

30 Jonasson F, Arnarsson A, Sasaki H, Peto T, Sasaki K, Bird AC. The prevalence of age-related maculopathy in Iceland. Arch Ophthalmol 2003; 121: 379-385.

31 Bressler NM, Munoz B, Maguire MG, Vitale SE, Schein OD, Taylor HR. et al. Five-year incidence and disappearance of drusen and retinal pigment epithelial abnormalities. Arch Ophthalmol 1995; 113: 301-308.

32 Soubrane G, Coscas G, Francais C, Koenig F. Occult subretinal new vessels in age-related macular degeneration. Natural History and early laser treatment. Ophthalmology 1990; 97: 649-657.

33 Stevens TS, Bressler NM, Maguire MG, Bressler SB, Fine SL, Alexander $\mathrm{J}$ et al. Occult choroidal neovascularisationin agerelated macular degeneration. A natural history study. Arch Ophthalmol 1997; 115: 345-350.
34 De Laey JJ. Natural history and prognosis of choroidal neovascularization. Doc Ophthalmol 1983; 55: 91-105.

35 Bressler SB, Pieramici DJ, Koester JM, Bressler NM. Natural history of minimally classic subfoveal choroidal neovascular lesions in the treatment of age-related macular degeneration with photodynamic therapy (TAP) investigation: outcomes potentially relevant to management - TAP report No. 6. Arch Ophthalmol 2004; 122: 325-329.

36 Grossniklaus HE, Gass JDM. Clinicopathologic correlations of surgically excised type 1 and type 2 submacular choroidal neovascular membranes. Am J Ophthalmol 1998; 126: 50-69.

37 Bynoe LA, Chang TS, Funata M, Del Priore LV, Kaplan HJ, Green WR. Histopathologic examination of vascular patterns in subfoveal neovascular membranes. Ophthalmology 1994; 101: 1112-1117.

38 Smith VC, Pokorny J, Diddie KR. Color matching and the Stiles-Crawford effect in observers with early age-related macular changes. J Opt Soc Am A 1988; 5 2113-2121.

39 Fitzgerald CR, Birch DG. Functional analysis of vision in patients after retinal detachment repair. Arch Ophthalmol 1980; 98: 1237-1244.

40 Elsner AE, Burns SA, Beausencourt E, Weiter JJ. Foveal cone photopigment distribution: small alterations associated with macular pigment distribution. Invest Ophthalmol Vis Sci 1998; 39: 2394-2404.

41 Elsner AE, Burns SA, Weiter JJ. Cone photopigment in older subjects: decreased optical density in early age-related macular degeneration. J Opt Soc Am A 2002; 19: 215-222.

42 Haritoglou C, Gandorfer A, Gass CA, Schaumberger M, Ulbig MW, Kampik A. The effect of indocyanine-green on functional outcome of macular pucker surgery. Am J Ophthalmol 2003; 135: 328-337.

43 Harbour JW, Smiddy WE, Flynn HW, Rubsamen PE. Vitrectomy for diabetic macular edema associated with a thickened and taut posterior hyaloid membrane. Am J Ophthalmol 1996; 121: 405-413.

44 Jonas JB, Kreissig I, Soefker A, Degenring RF. Intravitreal injection of triamcinolone for diffuse diabetic macular edema. Am J Ophthalmol 2003; 121: 57-61.

45 Age-Related Eye Disease Study Research Group. A randomized, placebo-controlled, clinical trial of high-dose supplementation with vitamins $\mathrm{C}$ and $\mathrm{E}$, beta carotene, and zinc for age-related macular degeneration and vision loss: AREDS report no. 8. Arch Ophthalmol 2001; 119: $1417-1436$

46 Reus NJ, Lemij HG. Scanning laser polarimetry of the retinal nerve fiber layer in perimetrically unaffected eyes of glaucoma patients. Ophthalmology 2004; 111: 2199-2203.

47 Mohammadi K, Bowd C, Weinreb RN, Medeiros FA, Sample PA, Zangwill LM. Retinal nerve fiber layer thickness measurements with scanning laser polarimetry predict glaucomatous visual field loss. Am J Ophthalmol 2004; 138: 592-601.

48 Greenfield DS, Knighton RW, Feuer WJ, Schiffman JC, Zangwill L, Weinreb RN. Correction for corneal polarization axis improves the discriminating power of scanning laser polarimetry. Am J Ophthalmol 2002; 134: 27-33.

49 Schlottman PG, De Cilla S, Greenfield DS, Caprioli J, Garway-Heath DF. Relationship between visual field 
sensitivity and retinal nerve fiber layer thickness as measured by scanning laser polarimetry. Invest Ophthalmol Vis Sci 2004; 45: 1823-1829.

50 Hunter DG, Patel SN, Guyton DL. Automated detection of foveal fixation by use of retinal birefringence scanning. Appl Opt 1999; 38: 1273-1279.

51 Misson GP. A Mueller matrix model of Haidinger's brushes. Ophthalmic Physiol Opt 2003; 23: 441-447.
52 Bone RA, Landrum JT. Macular pigment in Henle fiber membranes: a model for Haidinger's brushes. Vision Res 1984; 24: 103-108.

53 Naylor EJ, Stanworth A. The measurement and clinical significance of the Haidinger effect. Trans Opthal Soc UK 1955; 75: 67-79.

54 Hemenger RP. Dichroism of the macular pigment and Haidinger's brushes. J Opt Soc Am 1982; 72: 734-737. 